\title{
Overexpression of Tbx3 Predicts Poor Prognosis of Patients with Resectable Pancreatic Carcinoma
}

\author{
Hong-Cheng Wang, Qing-Cai Meng, Ze-Zhi Shan, Zhou Yuan, Xin-Yu Huang*
}

\begin{abstract}
Background: To determine the expressions of Tbx3, a member of subgroup belonging to T-box family, and its prognostic value in pancreatic carcinoma. Materials and Methods: We determined the expression levels of Tbx3 on both mRNA and protein levels in 30 pairs of fresh tumor tissues and paratumor tissues by quantitative real-time polymerase chain reaction (qRT-PCR) and Western blotting, respectively. In addition, protein level of Tbx 3 were identified using immunochemistry in 80 pairs of paraffin-embedded specimen. The correlations between Tbx3 expression and various clinicopathological parameters as well as overall survival were evaluated. Results: Tbx3 mRNA and protein levels in tumor tissues were significantly higher than in the paratumor tissues by qRT-PCR $(0.05 \pm 0.007$ vs. $0.087 \pm \mathbf{0 . 0 0 1}, \boldsymbol{p}<\mathbf{0 . 0 0 1})$ and western blotting $(\mathbf{1 . 1 3 4} \pm \mathbf{0 . 0 4 3} v s .0 .287 \pm \mathbf{0 . 0 1 7}, \boldsymbol{p}<\mathbf{0 . 0 0 1})$. The statistical analysis based on immunohistochemical evaluation suggested that Tbx3 aberrant expression was significantly associated with several conventional clinicopathological variables, such as gender, age, tumor position, preoperative CA19-9 level, pathological $\mathrm{T}$ staging and $\mathrm{N}$ staging. Univariate and multivariate analyses revealed that Tbx3 expression was an independent prognostic factor for overall survival $(<0.001)$. Conclusions: Our results suggest that overexpression of Tbx 3 is associated with poor prognosis of pancreatic cancer patients. However, additional clinical trials are needed to accurately validate this observation.
\end{abstract}

Keywords: Tbx3 - pancreatic carcinoma - prognosis

Asian Pac J Cancer Prev, 16 (4), 1397-1401

\section{Introduction}

Pancreatic carcinoma (PC) is a lethal malignancy, corresponding to the fourth leading cause of cancer death in world, with 5-year survival rates about 6\% (Hidalgo, 2010; Ferlay et al., 2013; Siegel et al., 2013). A majority of patients have been diagnosed with locally advanced or metastatic because of the lack of easily observable symptoms, and only $15 \%-20 \%$ of the patients have the chance for surgury and the rest could receive adjuvant therapies(Li et al., 2004). The prognosis for these patients remains poor, even though new antitumor drugs and techniques have been developed. Recently, much more attention has been paid to tumor molecules that were associated with PC prognosis (Ansari et al., 2011; Winter et al., 2013). Therefore, novel prognostic molecules should be identified to improve the outcome of patients with PC.

The transcription factor Tbx 3 belongs to the T-box family which is an ancient gene family important for organogenesis and embryogenesis, and recognizes a 20-24 nucleotide palindromic sequence called the T-site or half of the sequence (Muller and Herrmann, 1997; Minguillon and Logan, 2003). Tbx3 is widely expressed in the nervous system, mammary gland, skeleton, heart, eye, lungs and pancreas (Washkowitz et al., 2012). Haploinsufficiency of Tbx3 may cause ulnar-mammary syndrome (UMS), a disorder characterized by shortened limbs, heart abnormalities and defective mammary glands (Ballim et al., 2012). Furthermore, Tbx3 mutations have been linked to the pluripotency of embryonic stem cells and the invasiveness of cancer (Kim et al., 2008; Peres et al., 2010). It is overexpressed in many cancers including melanoma, bladder, mammary and liver cancers (Rowley et al., 2004; Lomnytska et al., 2006; Lyng et al., 2006; Renard et al., 2007).

Tbx3 is expressed in exocrine tissue in the postnatal, adult pancreas and tumor-derived exocrine cell lines (Washkowitz et al., 2012). Moreover, it is closely related to pancreatic lesions, such as solid pseudopapillary neoplasm (SPN), well-differentiated pancreatic neuroendocrine neoplasms (PENE) and PC (Hansel et al., 2004; Cavard et al., 2009; Begum and Papaioannou, 2011). In the present study, we examined the Tbx 3 expression at both mRNA and protein levels in PC samples, and analyzed the correlations between Tbx3 expressions and various clinicopathological parameters. Finally, we aimed to explore any possible associations with the prognosis of PC. 


\section{Materials and Methods}

\section{Patients and specimens}

Eighty primary tumor specimens with matched paratumor tissues were obtained consecutively from patients with pancreatic carcinoma (PC) undergoing surgery at the Department of General surgery, Shanghai Sixth People's Hospital Affiliated to Shanghai Jiaotong University. All specimens were determined as pancreatic ductal adenocarcinoma by pathological examinations. Among these patients, there were 47 males and 33 females, with age ranging from 27 to 87 years (mean, 63.5 years). Based on the TNM staging criteria from American Joint Committee on Cancer (AJCC), these tumor specimens include 38 stage I and 42 stage II. All patients with PC had been followed up for survival and outcome until Jan 2014. The clinicopathological parameters of the patients are shown in Table 1.

RNA extraction and quantitative real-time polymerase chain reaction

Total RNA was extracted using Trizol reagent (Invitrogen, USA) according to the manufacturer's instructions. Complementary DNA (cDNA) was generated using Superscript III Reverse Transcriptase

Table 1. The Correlation Between Tbx3 Expression and Clinicopathological Parameters in Patients With Pc

\begin{tabular}{|c|c|c|c|c|}
\hline \multirow{2}{*}{$\begin{array}{l}\text { Clinicopathological } \\
\text { parameters }\end{array}$} & \multirow[t]{2}{*}{$\mathrm{N}$} & \multicolumn{2}{|c|}{ Tbx3 expression } & \multirow[t]{2}{*}{$P$ value } \\
\hline & & High & Low & \\
\hline \multicolumn{5}{|l|}{ Gender } \\
\hline Male & 47 & 32 & 15 & 0.455 \\
\hline Female & 33 & 25 & 8 & \\
\hline \multicolumn{5}{|l|}{ Age } \\
\hline$\geq 60$ & 54 & 36 & 18 & 0.192 \\
\hline$<60$ & 26 & 21 & 5 & \\
\hline \multicolumn{5}{|l|}{ Tumor position } \\
\hline Head & 51 & 35 & 16 & 0.492 \\
\hline Body and tail & 29 & 22 & 7 & \\
\hline \multicolumn{5}{|c|}{ Preoperative CA19-9 level } \\
\hline Normal & 23 & 13 & 10 & 0.064 \\
\hline Elevated & 57 & 44 & 13 & \\
\hline \multicolumn{5}{|l|}{ Pathological T staging } \\
\hline T1-2 & 56 & 38 & 18 & 0.306 \\
\hline $\mathrm{T} 3$ & 24 & 19 & 5 & \\
\hline \multicolumn{5}{|c|}{ Pathological N staging } \\
\hline No & 27 & 15 & 12 & 0.027 \\
\hline N1 & 53 & 42 & 11 & \\
\hline
\end{tabular}

(Promega, USA). Expression of mRNA was determined by quantitative real-time polymerase chain reaction (qRT-PCR). $\beta$-actin was used as an internal control to normalize the amount of total RNA in each sample. The following primers were used for qRT-PCR analysis: Tbx3 sense, 5'-CCCGAAGAAGACGTAGAAGATGAC-3', Tbx 3 antisense 5'-C C GA A GA G AGGTGGAGGACGAC-3'; $\beta$-actin sense, 5'-CCTCCATCGTCCACCGCAAATG-3', $\beta$-actin antisense 5'-TGCTGTCACCTTCACCGTTCCA-3'. The PCR amplification consisted of 40 cycles $\left(95^{\circ} \mathrm{C}\right.$ for $15 \mathrm{~s}$ and $55^{\circ} \mathrm{C}$ for $\left.15 \mathrm{~s}\right)$ after an initial denaturation step $\left(95^{\circ} \mathrm{C}\right.$ for 10s). Relative expression of Tbx3 was calculated against $\beta$-actin mRNA level by using the $2^{-\Delta \Delta \mathrm{Ct}}$ method.

\section{Western blotting}

Total samples lysates were centrifuged at $12,000 \mathrm{~g}$ for 10 minutes at 4 . Then, the proteins were separated by $10 \%$ sodium dodecyl sulfate-polyacrylamide gel electrophoresis (SDS-PAGE) and transferred onto a polyvinylidene difluoride (PVDF) membranes. The membrane was incubated with primary antibodies against Tbx3 (1:1000, Abcam) at 4 overnight, washed with Tris-buffered saline with Tween 20 (TBST) 3 times, and incubated with HRP-labeled Goat Anti-Rabbit IgG (1:2000) for 2 hours at room temperature. The $\beta$-actin antibody (1:2000, Abcam) was used as internal control. The analysis of Western-blot result was performed by Image J 1.43 software.

\section{Immunohistochemical techniques}

Sections of paraffin-embedded tissue (4- $\mu$ m thick) were deparaffinaged with xylene and rehydrated with graded ethanol. Microwave heating was used to retrieve antigen. $0.3 \%$ hydrogen peroxide was subsequently added into the slides to block the endogenous peroxidase. Then, the slides were incubated with the primary antibody (dilution: $1: 300$ ) overnight at $4{ }^{\circ} \mathrm{C}$, followed by incubation of second antibody for 30 minutes. The slides incubated with PBS instead of primary antibody were applied as the negative control. Finally, all slides were stained with diaminobenzidine and then counterstained with hematoxylin.

\section{Immunohistochemical determination}

Two pathologists who were blinded to clinicopathological data performed staining evaluation,

Table 2. Significant Clinicopathological Parameters for Prognosis as Determined by Univariate and Multivariate Analysis

\begin{tabular}{|c|c|c|c|c|}
\hline \multirow[t]{2}{*}{ Clinicopathological parameters } & \multicolumn{2}{|c|}{ Univariate } & \multicolumn{2}{|c|}{ Munivariate* } \\
\hline & HR $(95 \% \mathrm{CI})$ & $P$ value ${ }^{\#}$ & HR $(95 \% \mathrm{CI})$ & $P$ value ${ }^{\#}$ \\
\hline Gender & $0.935(0.585-1.496)$ & 0.780 & & \\
\hline Age & $0.830(0.502-1.373)$ & 0.469 & & \\
\hline Tumor position & $0.867(0.531-1.415)$ & 0.567 & & \\
\hline Preoperative CA19-9 level & $0.609(0.360-1.031)$ & 0.065 & & \\
\hline Pathological T staging & $0.317(0.191-0.525)$ & $<0.001$ & $0.510(0.261-0.997)$ & 0.049 \\
\hline Pathological N staging & $0.385(0.237-0.627)$ & $<0.001$ & $0.517(0.270-0.991)$ & 0.047 \\
\hline Tbx3 expression & $0.523(0.308-0.889)$ & 0.017 & $0.522(0.303-0.902)$ & 0.020 \\
\hline
\end{tabular}

*HR, hazard ratio; CI, confidence interval; * Cox regression test; \# Significant difference $\mathrm{p}<0.05$ 
reaching a consensus after discussion when there was a controversy. Immunoreactivity for Tbx3 was evaluated by using a combined scoring system multiplying the staining intensity (SI) and the percentage of positive cells (PP). Scores from 0-3 were given for SI or PP as follows respectively: score of 0 , negative or $<5 \%$; score of 1 , weak or $6-25 \%$; score of 2 , moderate or $26-50 \%$; score of 3 , strong or $51-70 \%$ and score of 4 , only $71-100 \%$. Then, a final decision for $\mathrm{Tbx} 3$ expression was made according to the standard (low expression: score $<4$; high expression: score $\geq 4$ ).

\section{Statistical analysis}

All statistical analyses were performed using SPSS version 21.0 software (Chicago, IL). The $\chi^{2}$ test was used to determine the correlation between the expression of Tbx 3 and clinicopathologic parameters. Survival durations were calculated using the Kaplan-Meier method and intergroup difference was analyzed by the log-rank test. The Cox proportional hazards model was used for the univariate and multivariate analysis. For all analysis, a P value of less than 0.05 was considered to be statistically significant.

\section{Results}

Expression of Tbx3 in PC tissues and paratumor tissues The mRNA levels of Tbx 3 were determined in 30 fresh PC tissues and the corresponding paratumor control tissues using qRT-PCR. As shown in 'ure 1, Tbx3 mRNA levels in the tumor tissue was higher than in the control tissue and this difference was statistically significant $(0.056 \pm 0.007$ vs. $0.087 \pm 0.001, p<0.001)$. Furthermore, the expression of Tbx3 on protein levels was examined by Western blot analysis (Figure 2A and Figure 2B, 1.134 \pm 0.043 vs. $0.287 \pm 0.017, p<0.001)$. The expression of $\mathrm{Tbx} 3$ in 80 paraffin-embedded PC tissues were identified using immunochemistry, showing staining of Tbx3, among which 57 (71\%) were high expression and 23 (29\%) were lower expression. Detailed analysis revealed that the expression of Tbx 3 were mainly located in the cytoplasm of carcinoma cells. Representative immunohistochemical results were shown in Figure 3

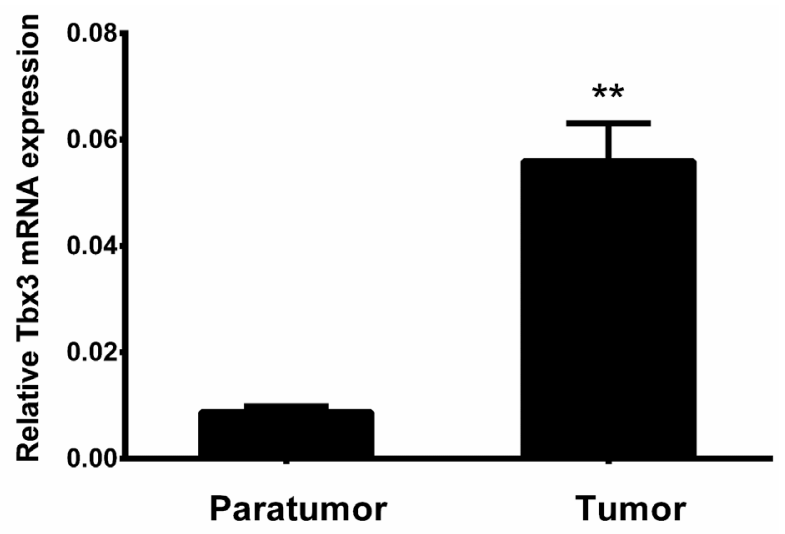

Figure 1. Tbx3 mRNA Levels in the Tumor Tissues was Higher than in the Corresponding Paratumor Control tissues and this Difference was Statistically Significant $(\mathbf{0 . 0 5 6} \pm \mathbf{0 . 0 0 7} v \boldsymbol{v s . 0 . 0 8 7 \pm 0 . 0 0 1 )}$. $* * p<0.001$ as Compared to the Control Group

\section{Clinicopathological association of Tbx3 expression}

Table 1 shows the association of Tbx 3 expression and clinicopathological characteristics. The overexpression of Tbx3 was significant associated with pathological $\mathrm{N}$ staging $(p=0.027)$ and no significant difference was observed in other clinicopathological parameters, including gender $(p=0.455)$, age $(p=0.192)$, tumor position (0.492), preoperative CA19-9 level $(p=0.064)$ and pathological T staging $(p=0.306)$.

\section{Prognostic significance of Tbx3 in PC after resection}

Kaplan-Meier survival analysis showed that pantients with high Tbx3 expression carried significantly poorer overall survival (OS) compared to patients with lower Tbx3 expression (Figure 4, $p=0.012$ ). Moreover, univariate analysis revealed OS of PC patients was significantly associated with pathological $\mathrm{T}$ staging $(p<0.001)$, pathological $\mathrm{N}$ staging tumor $(p<0.001)$ and Tbx 3 expression $(p=0.017)$. As exhibited in Table 2, Tbx3 expression, pathological $\mathrm{T}$ staging and pathological $\mathrm{N}$ staging ( $p=0.020,0.049,0.047$, respectively) could be
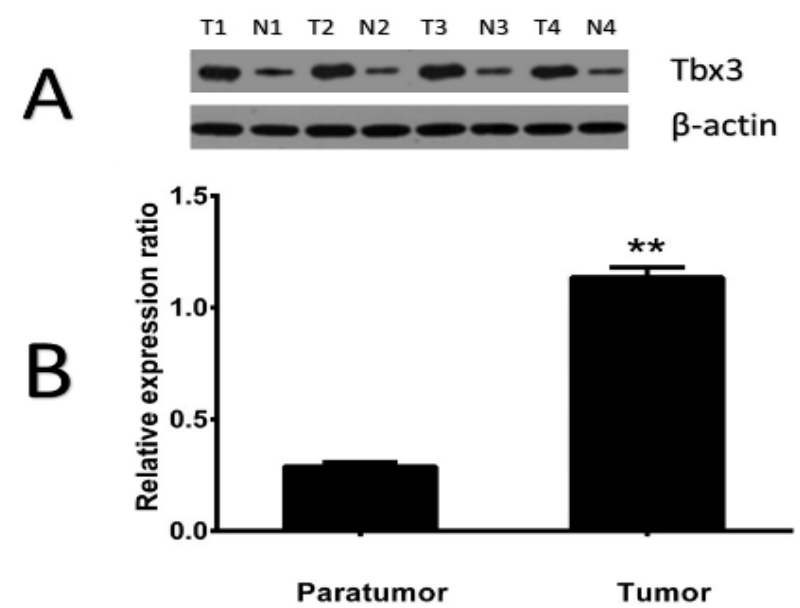

Figure 2. The Tbx3 Protein Levels from Tumor and Paratumor Tissues were Detected by Western Blot Analysis (1.134 \pm 0.043 vs. 0.287 \pm 0.017$)$. $\beta$-actin was used as Internal Control. $* * p<0.001$ as compared to the control group

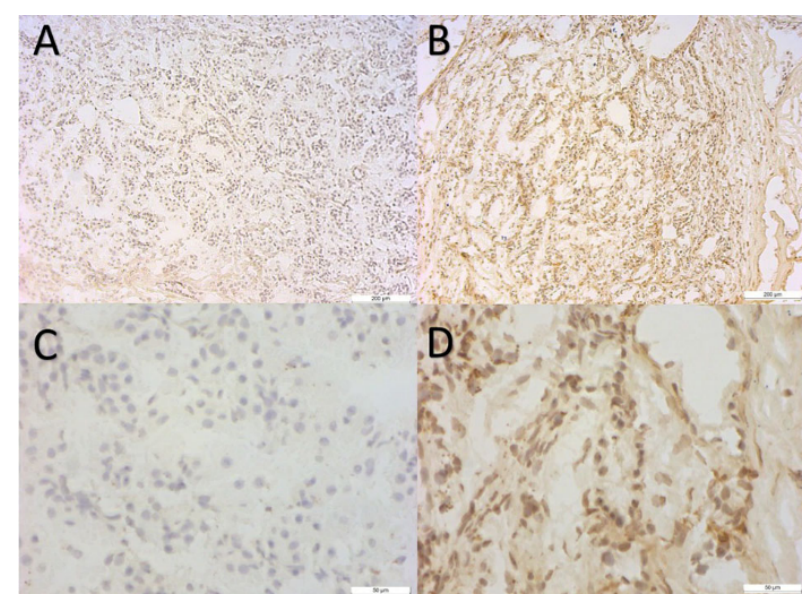

Figure 3. Representative Immunohistochemical Staining for Tbx3 in Tumor Tissues and Paratumor Tissues. Original magnification $\times 100$ : Tbx 3 expression in tumor tissues (A) and in paratumor tissues (B); Original magnification $\times 400$ : Tbx 3 expression in tumor tissues $(C)$ and in paratumor tissues (D) 


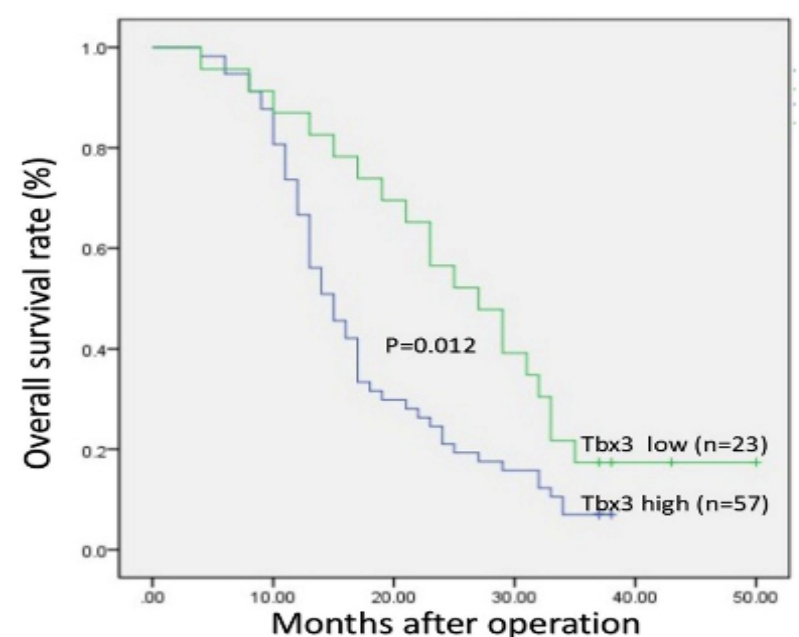

Figure 4. Tbx3 Expression Levels Significantly and Reversely Correlated with Overall Survival of Patients after Surgical Resection. The overall survival rate for PC patients with high expression of Tbx3 was lower than those with low expression (Log-rank test; $p=0.012$ )

identified as independent predictive factors in PC patients by multivariate Cox analysis.

\section{Discussion}

As is a member of the Tbx2 subfamily, Tbx 3 expression begins in the inner cell mass of the blastocyst and appears in the extraembryonic mesoderm during gastrulation. Moreover, it is expressed in many organs during normal mouse development (Chapman et al., 1996). Ito et al. revealed that Tbx 3 expression is related to cell proliferation and apoptosis in rat bladder both hyperplastic epithelial cells and carcinoma cells (Ito et al., 2005). Rodriguez et al. found that Tbx 3 may play a dual role in inhibiting senescence via repression of p21CIP1 expression, and enhancing melanoma invasiveness by decreasing E-cadherin levels (Rodriguez et al., 2008). In addition, accumulating evidence suggests that Tbx3 was also associated with clinicopathological features and poor prognosis in many malignant tumors (Renard et al., 2007; Rodriguez et al., 2008; Li et al., 2013; Du et al., 2014). However, its role in PC remains inconclusive. In the present study, we found that the Tbx 3 mRNA levels in tumor tissues was significantly higher than in paratumor tissues $(p<0.01)$. In addition to the mRNA level, we also examined the expression of Tbx 3 on the protein level. By western blot analysis and immunochemistry, we observed similar trends on $\mathrm{Tbx} 3$ protein levels as on its mRNA levels.

Further analysis on the correction between Tbx 3 expression levels and clinicopathological parameters revealed that high Tbx3 expression associated with tumor pathological $\mathrm{N}$ staging $(p=0.027)$, which may be linked with tumor invasion. Postoperative recurrences and metastasis are the main reasons for poor prognosis of PC patients. Perhaps TNM staging, especially N staging (infiltration range of regional lymph node) is considered as the key prognostic indicator for PC patients. More importantly, the outcome showed pantients with high Tbx3 expression carried significantly poorer prognosis compared to patients with lower Tbx3 expression performed by the Kaplan-Meier method as well as Cox multivariate proportional hazard model. Therefore, Tbx3 could be an independent predictive marker for PC patients.

Accumulating evidence suggested that Tbx3 contributes to tumorigenesis through interaction with several oncogenic pathways (Carlson et al., 2002; Rowley et al., 2004; Schmalhofer et al., 2009; Mowla et al., 2011). Fillmore et al. found that FGF signaling induces Tbx3 expression in normal mammary gland development and estrogen can increase Tbx 3 levels in breast cancer via FGF9-FGFR3 signaling (Fillmore et al., 2010). Upregulation of Tbx 3 can promote the bypass of senescence through inactivation of p53 via ARFMDM2-p53 tumor suppressor pathway (Howard and Ashworth, 2006; Zhang et al., 2011). Moreover, Yan et al. identified that Tbx3 can suppress GATA3, a downstream target of Tbx 3 , or alternatively regulate E-cadherin levels by binding to GATA3 and E-cadherin in breast cancer (Yan et al., 2010). Although the high Tbx3 expression is associated with the poor prognosis of many malignant tumors, the mechanism by which Tbx3 expression contributes to pancreatic tumorigenesis is still not clear and should be demonstrated by further study.

In summary, the correction between high Tbx3 expression and poor prognosis indicates its potential as a prognostic marker for PC patients. Further studies will be continued to investigate the tumorigenesis mechanism that Tbx 3 regulates in the development /metastasis of PC.

Ethics: This study was approved by the Ethics Committee of the Sixth People's Hospital Affiliated to Shanghai Jiao Tong University (Shanghai, China). Informed consent was obtained from each patient before tissue specimens were collected.

\section{Acknowledgements}

This work was funded by the Science and Technology Commission of Shanghai Municipality (NO.12DZ1940808), Shanghai, China and we thank all the patients and clinical investigators who were involved in this study.

\section{References}

Ansari D, Rosendahl A, Elebro J, et al (2011). Systematic review of immunohistochemical biomarkers to identify prognostic subgroups of patients with pancreatic cancer. Br J Surg, 98, 1041-55.

Ballim RD, Mendelsohn C, Papaioannou VE, et al (2012). The ulnar-mammary syndrome gene, $\mathrm{Tbx} 3$, is a direct target of the retinoic acid signaling pathway, which regulates its expression during mouse limb development. Mol Biol Cell, 23, 2362-72.

Begum S, Papaioannou VE (2011). Dynamic expression of Tbx2 and Tbx3 in developing mouse pancreas. Gene Expr Patterns, 11, 476-83.

Carlson H, Ota S, Song Y, et al (2002). Tbx3 impinges on the p53 pathway to suppress apoptosis, facilitate cell transformation and block myogenic differentiation. Oncogene, 21, 3827-35.

Cavard C, Audebourg A, Letourneur F, et al (2009). Gene expression profiling provides insights into the pathways involved in solid pseudopapillary neoplasm of the pancreas. 
J Pathol, 218, 201-9.

Chapman DL, Garvey N, Hancock S, et al (1996). Expression of the T-box family genes, Tbx 1-Tbx5, during early mouse development. Dev Dyn, 206, 379-90.

Du HF, Ou LP, Yang X, et al (2014). A new PKCalpha/beta/ TBX3/E-cadherin pathway is involved in PLCepsilonregulated invasion and migration in human bladder cancer cells. Cell Signal, 26, 580-93.

Ferlay J, Steliarova-Foucher E, Lortet-Tieulent J, et al (2013). Cancer incidence and mortality patterns in Europe: estimates for 40 countries in 2012. Eur J Cancer, 49, 1374-403.

Fillmore CM, Gupta PB, Rudnick JA, et al (2010). Estrogen expands breast cancer stem-like cells through paracrine FGF/ Tbx3 signaling. Proc Natl Acad Sci U S A, 107, 21737-42.

Hansel DE, Rahman A, House M, et al (2004). Met protooncogene and insulin-like growth factor binding protein 3 overexpression correlates with metastatic ability in welldifferentiated pancreatic endocrine neoplasms. Clin Cancer Res, 10, 6152-8.

Hidalgo M (2010). Pancreatic cancer. N Engl J Med, 362, 1605-17.

Howard B, Ashworth A (2006). Signalling pathways implicated in early mammary gland morphogenesis and breast cancer. PLoS Genet, 2, 112.

Ito A, Asamoto M, Hokaiwado N, et al (2005). Tbx3 expression is related to apoptosis and cell proliferation in rat bladder both hyperplastic epithelial cells and carcinoma cells. Cancer Lett, 219, 105-12.

Kim J, Chu J, Shen X, et al (2008). An extended transcriptional network for pluripotency of embryonic stem cells. Cell, 132, 1049-61.

Li D, Xie K, Wolff R, et al (2004). Pancreatic cancer. Lancet, 363, 1049-57.

Li J, Weinberg MS, Zerbini L, et al (2013). The oncogenic TBX3 is a downstream target and mediator of the TGF-beta1 signaling pathway. Mol Biol Cell, 24, 3569-76.

Lomnytska M, Dubrovska A, Hellman U, et al (2006). Increased expression of cSHMT, Tbx3 and utrophin in plasma of ovarian and breast cancer patients. Int J Cancer, 118, 412-21.

Lyng H, Brovig RS, Svendsrud DH, et al (2006). Gene expressions and copy numbers associated with metastatic phenotypes of uterine cervical cancer. BMC Genomics, $\mathbf{7}$, 268.

Minguillon C, Logan M (2003). The comparative genomics of T-box genes. Brief Funct Genomic Proteomic, 2, 224-33.

Mowla S, Pinnock R, Leaner VD, et al (2011). PMA-induced up-regulation of TBX3 is mediated by AP- 1 and contributes to breast cancer cell migration. Biochem J, 433, 145-53.

Muller CW, Herrmann BG (1997). Crystallographic structure of the T domain-DNA complex of the Brachyury transcription factor. Nature, $\mathbf{3 8 9}$, 884-8.

Peres J, Davis E, Mowla S, et al (2010). The Highly Homologous T-Box Transcription Factors, TBX2 and TBX3, Have Distinct Roles in the Oncogenic Process. Genes Cancer, 1, 272-82.

Renard CA, Labalette C, Armengol C, et al (2007). Tbx3 is a downstream target of the Wnt/beta-catenin pathway and a critical mediator of beta-catenin survival functions in liver cancer. Cancer Res, 67, 901-10.

Rodriguez M, Aladowicz E, Lanfrancone L, et al (2008). Tbx3 represses E-cadherin expression and enhances melanoma invasiveness. Cancer Res, 68, 7872-81.

Rowley M, Grothey E, Couch FJ (2004). The role of Tbx2 and Tbx3 in mammary development and tumorigenesis. $J$ Mammary Gland Biol Neoplasia, 9, 109-18.

Schmalhofer O, Brabletz S, Brabletz T (2009). E-cadherin, beta-catenin, and ZEB1 in malignant progression of cancer.
Cancer Metastasis Rev, 28, 151-66.

Siegel R, Naishadham D, Jemal A (2013). Cancer statistics, 2013. CA Cancer J Clin, 63, 11-30.

Washkowitz AJ, Gavrilov S, Begum S, et al (2012). Diverse functional networks of Tbx3 in development and disease. Wiley Interdiscip Rev Syst Biol Med, 4, 273-83.

Winter JM, Yeo CJ, Brody JR (2013). Diagnostic, prognostic, and predictive biomarkers in pancreatic cancer. $J$ Surg Oncol, 107, 15-22.

Yan W, Cao QJ, Arenas RB, et al (2010). GATA3 inhibits breast cancer metastasis through the reversal of epithelialmesenchymal transition. J Biol Chem, 285, 14042-51.

Zhang JF, He ML, Qi D, et al (2011). Aqueous extracts of Fructus Ligustri Lucidi enhance the sensitivity of human colorectal carcinoma DLD-1 cells to doxorubicin-induced apoptosis via Tbx3 suppression. Integr Cancer Ther, 10, 85-91. 